\title{
Harnessing Systems Engineering Methodology in Using Earth Science Research Data for Real Applications
}

\author{
Shahid Habib ${ }^{1}$, Fritz S. Policelli ${ }^{2}$, Vicki M. Zanoni ${ }^{2}$ \\ ${ }^{\text {I}}$ Earth Sciences Directorate, NASA, Goddard Space Flight Center, Greenbelt, Maryland 20771 \\ ${ }^{2}$ Earth Science Applications Directorate, NASA, Stennis Space Flight Center, Bay Saint Louis, Mississippi 39529
}

\begin{abstract}
For the last three decades, Earth science remote sensing technologies have been providing an enormous amount of useful data and information serving to broaden our understanding of the home planet as a system. NASA's Earth science program has deployed about 18 complex satellites and is in the process of defining and launching multiple observing systems in this decade. At the same time, the European Community and many other countries such as Russia, France, India, Japan, and China have also significantly contributed to Earth science research. To date, the majority of such efforts have concentrated on expanding our scientific understanding of the multiple nonlinear and chaotic processes of Earth's behavior. In recent years, legislators and stakeholders have put serious pressure on the science community to devote more attention to making use of scientific results for societal benefit. For instance, there are a number of areas such as energy forecasting, aviation safety, agricultural efficiency, disaster management, air quality and public health that can directly take advantage of Earth science results to analyze and predict large scale problems and conditions. This is becoming even more important now that we live in a global economy interconnected via the internet and transportation systems; regional environmental conditions can have far reaching impact across continental boundaries. These factors dictate requirements for global data that can help us assess and control the devastating problems of famine, water resources, wildfires, human health and more. To do this requires a serious, organized, and systematic approach that transfers fundamental research products to the applied sciences domain. This paper presents a systems engineering and management process that can effectively make such transfer of data to the user community. Examples are presented on how the above decision making framework can help in solving critical problems such as the spread of vector borne diseases, forecasts of harmful algal blooms as well as forest fires and wildfires, and the intercontinental transport of dust storms and pollution.
\end{abstract}

\section{INTRODUCTION}

Remote sensing technology and industry have come a long way in the past 40 years. The major catalyst has been the government sector. Civilian Government agencies such as NASA (National Aeronautics and Space Administration), ESA (European Space Agency), JAXA (Japan Aerospace Exploration Agency), FSA (Federal Space Agency of Russia), CNES (Centre National d'Etudes Spatiale) and others have taken advantage of such technologies for basic research in both Earth and Space science. For example, over the past 30 years, the Landsat program has provided a rich heritage of multispectral remote sensing data of the Earth's surface. Since the early days of TIROS and Landsat, increasingly sophisticated satellites have been launched with a diverse set of sensors that measure atmospheric temperature, humidity, ocean altimetry, ocean color, ozone, aerosols, and many more atmospheric constituents affecting our biosphere. Land imaging satellites such as Landsat and Spot have provided a wealth of information in mapping the entire globe in VIS and IR (visible and infrared spectral band) at a resolution of 10-30 meters. This has provided a large volume of data to the science and the user communities for interpreting the entire globe in terms of biodiversity, land cover and land use changes. However, higher resolution was still needed for more detailed work related to urban planning and disaster aftermath analysis. Commercial satellites such as IKONOS and QuikBird have lately filled this void with their higher resolution $(1 \mathrm{~m})$ imagery.

This has brought the remote sensing technologies to a crossroads: who should continue to invest in this arena? Should the Government entities still be in the driver seat or should they relinquish their leadership and pass the baton on to the private sector? There are two fundamental issues related to this subject:

(1) What is the scientific value in continuing this area of exploration? The simple answer is that the study of the planet Earth is still in its early stages. The nature of Earth science research is such that it generates more questions than answers. Additional data, models, and analyses are required to further understand the physics of the problems in question.

(2) The other issue is: how can we best link space-based investments to near-term societal benefits? This issue clearly involves a trade-off with achieving near-term scientific results.

Based on the present economic environment, one has to address the above problems methodically. The planning for future Earth observing sensors should default to an economic justification process before initiating any major mission [1]. Therefore, one has to think much more broadly in terms of 'Science for Society.' This requires a cohesive and well thought-out process to transition the research results to the operational community for use in their decision making tools. For example, global precipitation records may not be directly adaptable to weather forecasting models. Conversely, the operational measurements of the equatorial precipitation record may not be a good fit for the scientific research. Therefore, such transition of research products must begin with an understanding of the operational requirements. 


\section{SCIENCE PRODUCTS AND DECISION MAKING}

Traditionally, to date, most of the decision making tools have been at regional to local levels and have relied on some level of automation and near real time data input. However, with the advent of satellite data and global science models, more realistic boundary conditions are available and can be used to improve the accuracy of initial conditions for regional models. This is particularly true when, for example, one is analyzing the local air quality and developing a reasonable prediction for the next 24 to 48 hours. This is relatively achievable when tropospheric ozone is in question; however, the problem becomes rather nontrivial when other airborne particles and aerosols are taken into consideration. There is not a sound scientific way to analyze, segregate and estimate the aerosols in a parcel or volume of air. Similarly, while predicting the time and path of a tornado well in advance is a monumental task and nearly impossible, global and regional observations and weather models can provide a warning that conditions indicate a high probability of tornado formation.

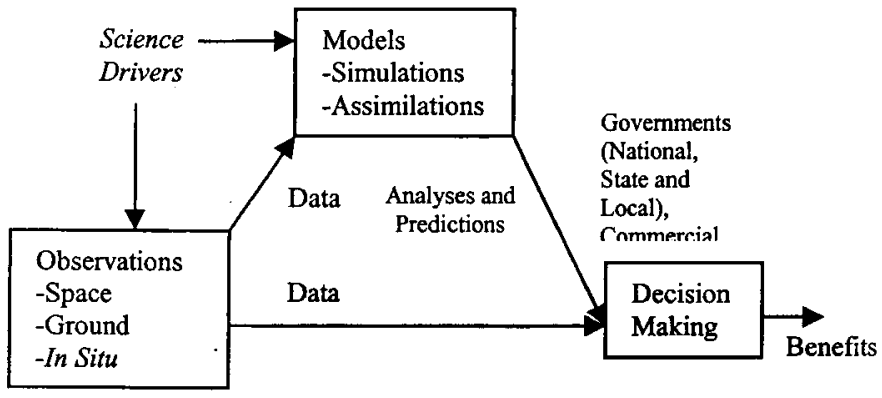

Fig. 1. Linking observations to models to decision making.

The decision making process at any level can use various tools that feed into some type of decision support system (either manual or computerized) in order to make calculated in situ measurements. To summarize this integrated systems architecture, the observations provide data and information; scientific models use these data and produce predictive results that help in reducing potential uncertainties inherent to a variety of Earth processes such as weather, climate variability, or volcanic eruptions. The essential first step in tying all of these elements of this architecture together is to bring the user community on board and to understand and crisply document their needs in terms of type and characteristics of data/products. This requires a comprehensive systems engineering and management process to effectively integrate the science, engineering and management players to solve such societal problems.

\section{SYSTEMS ENGINEERING PROCESS}

In any results-oriented environment, it is important to follow an approach that evaluates and documents requirements, provides the necessary material and products for verification, and finally benchmarks the improvement to decision making realized by the use of new data/ products. In the Applications arena it is deemed even more necessary to adapt such an approach. The overall goal for Earth Science Applications projects is to benchmark the benefit of relevant Earth science data and products within the user community. Therefore, it is essential that there be a user with his/her decision making system-a decision support system (DSS) - who is willing to incorporate science data into his/her decision making process.

The first step is to find a decision support system (DSS) and identify its owner organization. This requires close coordination of and dialogue between the science and the user communities to ensure that the data/product provider and the data/product receiver develop a clear understanding of what to expect from each other. The overall process as shown in Fig. 2 is simple, straightforward and designed to achieve successful results. The structure provides

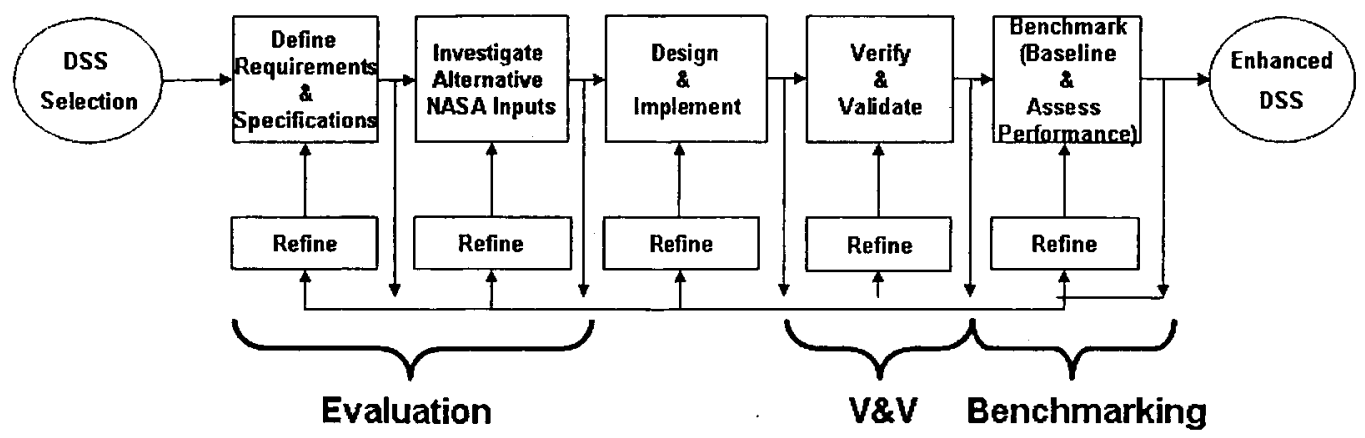

Fig. 2. Systems engineering life cycle covering the definition, development, test and verification and commissioning phase indicated here as benchmarking.

decisions. Therefore, depending upon the decision level, these decision support systems can be at a global, regional, or individual scale. Fig. 1 presents an overall architecture [2] that communicates the integration of specific observations and models into the decision making tool(s). This fundamental concept can be applicable to space, terrestrial or checkpoints that allow for the early identification of mismatches or false expectations so that long-term risks can be avoided. The basic principles of Systems Engineering should lead to scalable, systemic and sustainable solutions and processes, which in turn contribute to the success of the mission, goals, and objectives of each Application. The 
process is iterative and requires continuous coordination and dialogue among all team members. The basic phases are described below [3]:

- Evaluation involves understanding the requirements for and technical feasibility of Earth science and remote sensing tools and methods for addressing DSS needs.

- Verification and Validation $(V \& V)$ includes measuring the performance characteristics of data, information, technology, and/or methods, and assessing the ability of these tools to meet the requirements of the DSS.

- Benchmarking is the incorporation of NASA inputs into an operational DSS and the subsequent documentation of the resulting impacts and outcomes.

\section{NATIONAL APPLICATIONS IMPLEMENTATION}

NASA is taking the systems engineering approach in defining and transferring its Earth Science research data and products to the user and operational communities. There are twelve carefully selected Applications: Air Quality, Invasive Species, Water Management, Coastal Zone, Public Health, Disaster, Homeland Security [4], Aviation Safety, Energy, Agriculture Efficiency, Ecological Forecasting, and Carbon Management. Most of these Applications have multiple

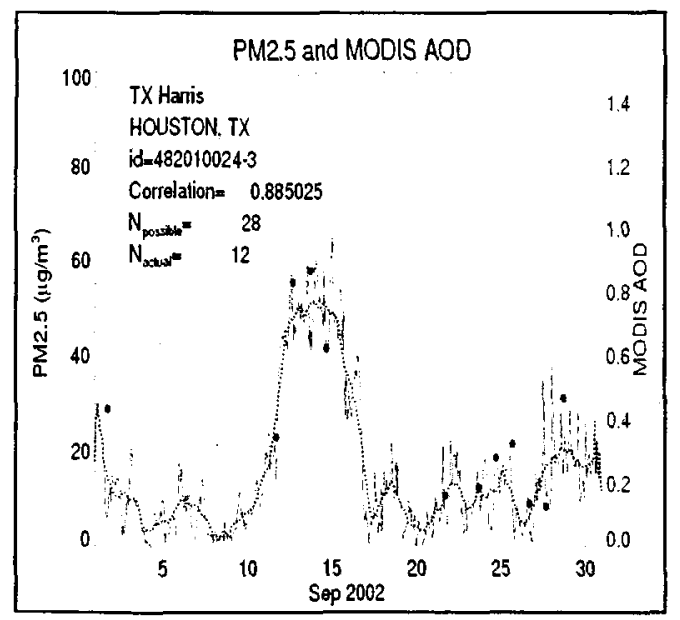

Fig.3. Time series of ground measurements is correlated with space based observations as indicated by dots on the graph (courtesy of $L$. Reimer and A. Chu of NASA/GSFC).

projects under them and are currently under development. The level of maturity for all of these projects varies tremendously in part due to the difficulty of identifying corresponding decision support systems and/or the slow pace of incorporation of science data into relevant products. However, each Application project is required to follow the systems engineering methodology and process as described above. In Applications projects, experience has shown that it is very important to sensitize the science community to the importance of following a systems engineering approach.
One of the projects recently completed deals with The U.S. Environmental Protection Agency's (EPA) Air Quality Index Decision Support System as part of the Air Quality Application. The main objective of this project was to work with EPA regional air quality assessment team to determine the air quality in major US cities. Presently, the EPA makes multiple in situ measurements using field based sun photometers to determine the optical depth of incoming sunlight. Based on the absorption index, the EPA [5] can determine the particulates present in the air sample. Small ( $2.5 \mu \mathrm{m}$ or smaller) particulate size is of paramount interest because particles of this size can be inhaled easily and can contribute to various health problems such as asthma. This EPA assessment can now be enhanced using the science data acquired from NASA's Moderate Resolution Imaging Spectroradiometer (MODIS) direct broadcast channel. This capability provides a spatial coverage of up to $2200 \mathrm{~km}$ using its 36 discrete bands ranging from ultraviolet (UV) to visible (VIS) to infrared (IR) bands. NASA has developed an algorithm that correlates the aerosol optical thickness obtained from MODIS measurements with the EPA's ground based in situ sensors (Fig. 3).

\section{SUMMARY}

Experience has shown that it is a challenging task to utilize science products for societal benefits. A paradigm shift is required to encourage the science community to think about the Applications areas as well as the fundamental Earth science research. However, this can be expedited if the user and scientific communities work together to understand the requirements and avail themselves of support and processes provided by the systems engineering community. NASA has adapted this approach to ensure the successful and smooth transition of science products to societal benefits. In the long run, a sensor web [6] approach could be more effective in providing both the temporal and spatial coverage so that real time results could reach each individual citizen in a timely manner in order to influence his/her daily life decisions.

\section{REFERENCES}

[1] S. Habib, "Technology Thrust for Future Earth Science Application," Optical Remote Sensing of the Atmosphere and Clouds, Second SPIE Asia-Pacific Symposium, Sendai, Japan, October 9-12, 2000.

[2] S. Habib and N..Nokra, "Transferring Knowledge from Observations and Models to Decision Makers," SPIE Europe, Barcelona, Spain, September 12-16, 2003.

[3] NASA, "Evaluation and Enhancement of Decision Support Tools FY2003 Report," Earth Science Applications Directorate, John C. Stennis Space Center, Mississippi, 2003.

[4] S. Habib, D. Zukor and S. Ambrose, "Using Science Driven Technologies for Defense and Security Applications," SPIE Defense and Security Symposium, Orlando, Florida, April 12-16, 2004.

[5] D. A. Chu, Y. J. Kaumfan, et. al., "Global monitoring of air pollution over land from the Earth Observing System-Terra Moderate Resolution Imaging Spectroradioimeter (MODIS)" Geophys. Res., 108(D21), $10.1029 / 2002 J D 003179,2003$.

[6] S. Habib and S. Talabac, "Space-based Sensor Web for Earth Science Applications - An Integrated Architecture for Providing Societal Benefits," ISPRS, Istanbul, Turkey, July 12-23, 2004. 